Document downloaded from:

http://hdl.handle.net/10251/78057

This paper must be cited as:

Valero Bresó, A.; Sahuquillo Borrás, J.; Petit Martí, SV.; Duato Marín, JF. (2013). Exploiting Reuse Information to Reduce Refresh Energy in On-Chip eDRAM Caches. ACM. doi:10.1145/2464996.2467278.

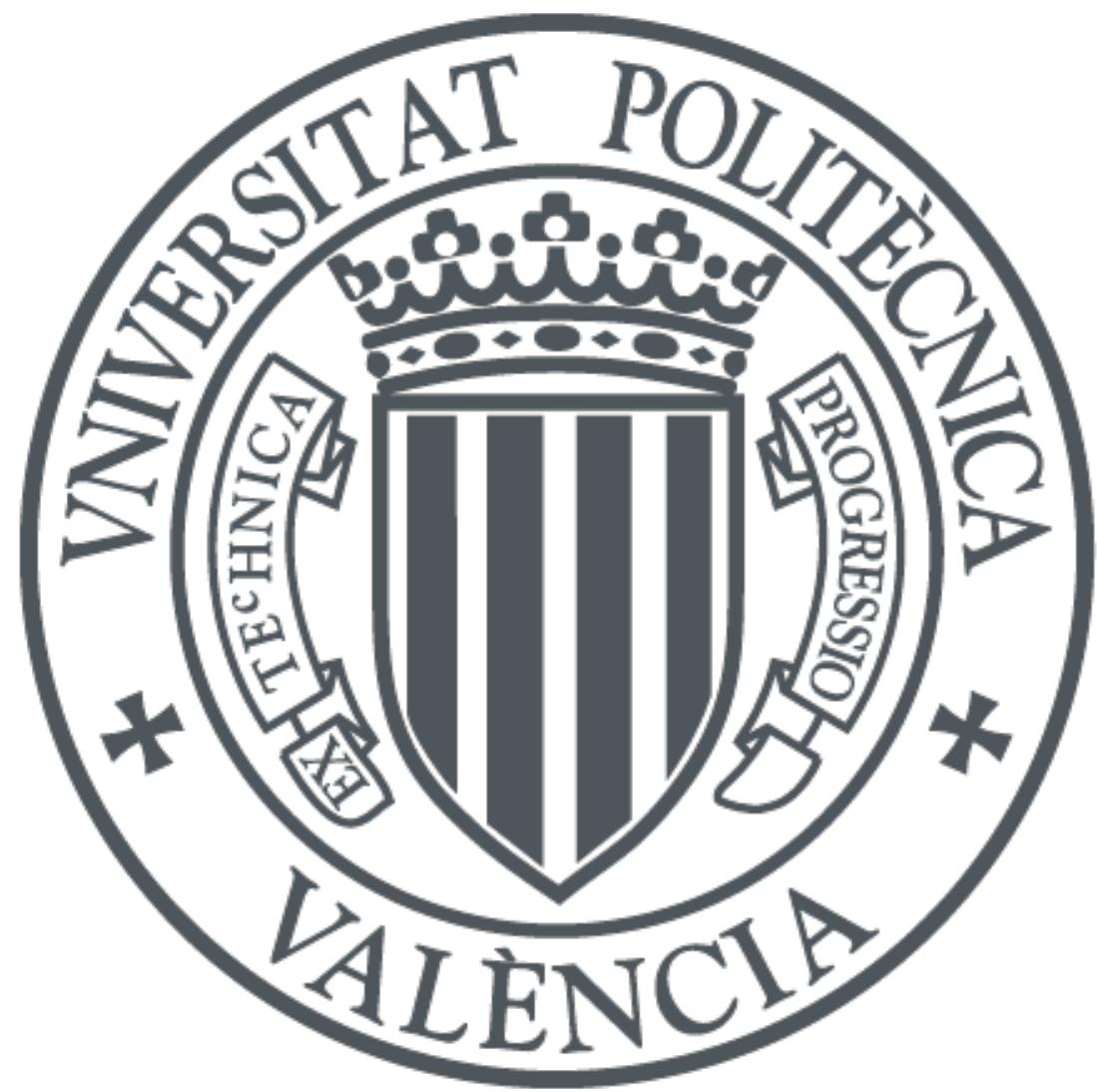

The final publication is available at

http://dx.doi.org/10.1145/2464996.2467278

Copyright ACM

Additional Information

(C) Owner/Author 2013. This is the author's version of the work. It is posted here for your personal use. Not for redistribution. The definitive Version of Record was published in ICS '13 Proceedings of the 27th international ACM conference on International conference on supercomputing; http://dx.doi.org/10.1145/2464996.2467278. 


\title{
Exploiting Reuse Information to Reduce Refresh Energy in On-Chip eDRAM Caches
}

\author{
Alejandro Valero, Julio Sahuquillo, Salvador Petit, and José Duato \\ Department of Computer Engineering \\ Universitat Politècnica de València \\ Valencia, Spain \\ alvabre@gap.upv.es, \{jsahuqui, spetit, jduato\}@disca.upv.es
}

\begin{abstract}
This work introduces a novel refresh mechanism that leverages reuse information to decide which blocks should be refreshed in an energy-aware eDRAM last-level cache. Experimental results show that, compared to a conventional eDRAM cache, the energy-aware approach achieves refresh energy savings up to $71 \%$, while the reduction on the overall dynamic energy is by $65 \%$ with negligible performance losses.
\end{abstract}

\section{Categories and Subject Descriptors}

B.3.2 [Design Styles]: Cache memories

\section{Keywords}

MRU-Tour; on-chip caches; selective refresh

\section{PROPOSED APPROACH}

Refresh operations in on-chip eDRAM caches incur in a significant fraction of the total dynamic energy consumed by these memories. Prior works have addressed this energy overhead by reducing the impact of inter-cell variability on refresh energy [3].

Unlike these proposals, this work pursues to minimize the number of refresh operations by applying selective refresh in an energyaware eDRAM last-level (L2) cache. The proposal aims to avoid refreshing useless lines in order to save energy and prevent performance losses. The devised refresh policy exploits reuse information to decide whether a cache block should be refreshed. To this end, the proposal works on the MRU-Tour (MRUT) concept [2], which is referred to as the number of times that a block enters in the MRU position of the LRU stack. Based on the observation that most blocks in L2 caches exhibit a single MRUT at the time they are evicted, the refresh mechanism does not periodically refresh the target block if it has only one MRUT.

To save energy, the proposed energy-aware cache only accesses in a first stage the tag array and a predicted cache bank in the data array. If the requested block is not stored in the predicted bank, then the target bank is accessed in a second stage. This mechanism always predicts the same physical bank, and MRU blocks are stored in that bank by performing data movements between ways similar to as done in [1]. Each bank implements two cache ways.

\section{EXPERIMENTAL RESULTS}

The proposed cache has been modeled with SimpleScalar and CACTI to obtain performance and energy consumption, respectively, for SPEC benchmarks.

Copyright is held by the author/owner(s). ACM X-XXXXX-XX-X/XX/XX.

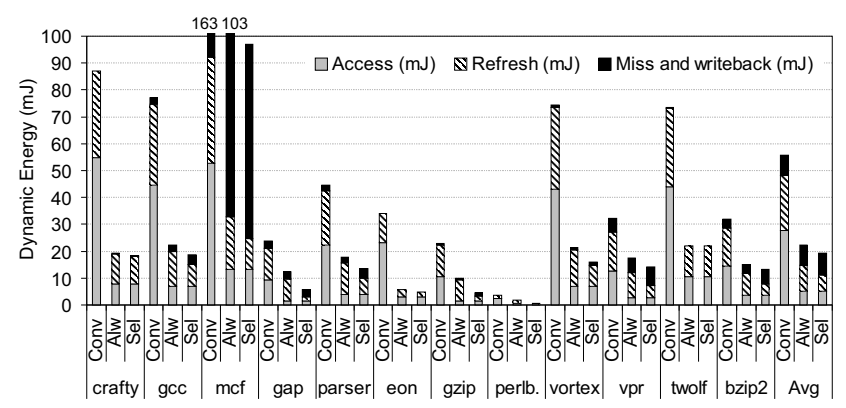

Figure 1: Dynamic energy consumption (in $\mathbf{m J}$ ).

Figure 1 plots the dynamic energy of a 2MB-16way L2 cache organization classified into Access, Refresh, and Miss and writeback energy. The latter covers the expenses of accessing to a $2 \mathrm{~GB}$ DRAM main memory. The energy consumption of the data movements between ways has been taken into account in the Access category. Label Conv refers to a conventional eDRAM cache that accesses in parallel all the tags and all the banks and uses a conventional refresh policy. Alw and $\mathrm{Sel}$ refer to the conventional and selective policies, respectively, both applied in the energy-aware scheme.

Compared to Conv, both Access and Refresh energy are largely reduced by the energy-aware scheme mainly because it accesses first just the MRU bank. As observed, Sel reduces the refresh consumption with respect to $A l w$, and it compensates the increase in the Miss and writeback energy caused by requests to non-refreshed blocks. Overall, the refresh savings of $\mathrm{Sel}$ are on average by $71 \%$ with respect to Conv. This percentage is by $65 \%$ when the whole energy is considered. These benefits come with minimal performance degradation (by $1.3 \%$ on average) with respect to Conv.

\section{ACKNOWLEDGMENTS}

This work was supported by the Spanish Ministerio de Economía y Competitividad (MINECO) and Plan E funds, under Grants TIN2009-14475-C04-01 and TIN2012-38341-C04-01.

\section{REFERENCES}

[1] J. Lira et al. Implementing a hybrid SRAM/eDRAM NUCA architecture. In Proc. 18th Int'l Conf. High Perform. Comput., pages 1-10, 2011.

[2] A. Valero et al. Combining Recency of Information with Selective Random and a Victim Cache in Last-Level Caches. ACM Trans. Arch. Code Opt., 9(3):16:1-16:20, 2012.

[3] C. Wilkerson et al. Reducing Cache Power with Low-Cost, Multi-bit Error-Correcting Codes. In Proc. 37th Int'l Symp. Comput. Arch., pages 83-93, 2010. 